\title{
IJCV Special Issue on 3D Object Retrieval
}

\author{
Foreword by the Guest Editors
}

\author{
Theoharis Theoharis • Ioannis Pratikakis • \\ Michela Spagnuolo
}

Received: 2 November 2009 / Accepted: 5 November 2009 / Published online: 24 November 2009

(C) Springer Science+Business Media, LLC 2009

3D object representations have become an integral part of modern computer graphics applications, such as computeraided design, game development and film production. At the same time, 3D data have become very common in domains such as computer vision, computational geometry, molecular biology and medicine. The rapid evolution in graphics hardware and software development, in particular the availability of low cost 3D scanners, has greatly facilitated 3D model acquisition, creation and manipulation, giving the opportunity to experience applications using 3D models to a large user community. As the number of 3D models is rapidly growing, the development of efficient search mechanisms is required for the effective retrieval of 3D objects from large repositories, both of a single class (such as human faces) and across classes. As this field gains momentum, a number of dedicated events appear within well-known conferences; these include a series of Workshops on 3D Object Retrieval (3DOR) within the Eurographics conference since 2008, along with the IEEE Workshop on Search in 3D at

\section{T. Theoharis}

Department of Informatics \& Telecommunications,

University of Athens, Panepistimioupolis, 15784 Athens, Greece

e-mail: theotheo@di.uoa.gr

I. Pratikakis ( $\square)$

Institute of Informatics and Telecommunications, National Center for Scientific Research “Demokritos", P. Grigoriou \& Neapoleos, 15310 Agia Paraskevi, Athens, Greece

e-mail: ipratika@iit.demokritos.gr

\section{Spagnuolo}

Istituto per la Matematica Applicata e le Tecnologie Informatiche, Consiglio Nazionale delle Ricerche, Via De Marini, 6,

16149 Genova, Italy

e-mail: spagnuolo@ge.imati.cnr.it
CVPR'08 as well as its successor, the IEEE Workshop on Search in 3D and Video at ICCV'09.

The aim of this special issue is fully compatible with the spirit of 3DOR events which was to stimulate researchers from different fields (computer vision, computer graphics, machine learning and human-computer interaction) who work on the common goal of 3D object retrieval, to present high caliber state-of-the-art work in the field and thus, provide a cross-fertilization ground that will stimulate discussions on the next steps in this important research area.

55 papers were submitted to the Special Issue, of which 16 were accepted after a rigorous review process. The topics covered by the Special Issue represent the key current research issues in 3D Object Retrieval and its state-of-theart. These include pose determination, robust shape descriptors for generic collections using monomodal or multimodal queries, isometry-invariant and non-rigid shape matching, hierarchical shape segmentation and registration, retrieval from sub-part queries, retrieval from complex scenes containing clutter and occlusions, shape similarity for $3 \mathrm{D}$ video sequences as well as learning schemes for similarity measures using relevance feedback and supporting partial shape retrieval.

We would like to thank the large number of reviewers involved, recognized experts in the field, since their input determined the outcome of the selection and revision processes. Also the authors themselves for their patience and for submitting significant contributions to this Special Issue. And last but not least, the Editorial Office of Springer for their continued support and the IJCV Editors involved, Christoph Schnörr and Katsushi Ikeuchi, for resolving issues that arose throughout the long process.

Guest Editors 